(Submitted to: the Journal of Materials and Manufacturing Processes \& TMS 2003 Annual Meeting, Session: Materials Processing under the Influence of Electric and Magnetic Fields)

\title{
FLUID FLOW AND SOLIDIFICATION UNDER COMBINED ACTION OF MAGNETIC FIELDS AND MICROGRAVITY
}

\author{
B. Q. Li*, Y. Shu and K. Li \\ Washington State University \\ Pullman, WA 99163
}

and

H. C. de Groh

NASA Glenn Center

Cleveland, $\mathrm{OH} 44135$

September, 2002

\footnotetext{
- To whom correspondence is made.
} publication. Because of changes that may be made before formal publication, this preprint is made available with the understanding that it will not be cited or reproduced without the permission of the author. 


\begin{abstract}
Mathematical models, both 2-D and 3-D, are developed to represent g-jitter induced fluid flows and their effects on solidification under combined action of magnetic fields and microgravity. The numerical model development is based on the finite element solution of governing equations describing the transient g-jitter driven fluid flows, heat transfer and solutal transport during crystal growth with and without an applied magnetic field in space vehicles. To validate the model predictions, a ground-based g-jitter simulator is developed using the oscillating wall temperatures where timely oscillating fluid flows are measured using a laser PIV system. The measurements are compared well with numerical results obtained from the numerical models. Results show that a combined action derived from magnetic damping and microgravity can be an effective means to control the melt flow and solutal transport in space single crystal growth systems.
\end{abstract}




\section{Introduction}

During solidification processing of materials under terrestrial environment, the imposed temperature gradients give rise to a circulating natural convection, which may affect the thermal distribution and solutal distribution in the liquid pool. The latter finally ends in the solidified materials, causing unwanted impurity striation and other defects [1]. Space environment, in which gravity is significantly reduced, provides a useful means to suppress this convection and thereby providing an opportunity to grow crystals in a diffusion-controlled regime. While the enthusiasm of going to space has been cherished by some flight experiments in which high quality crystals are obtained, it has been largely quenched by others in which poor quality crystals are produced. This inconsistency in space grown crystal qualities is found to be attributed to the existence of perturbed gravity forces in space, or g-jitter as often referred to in literature, which are random in nature and varies both spatially and temporally [2].

Gravity perturbation is not detected on earth. It is solely associated with microgravity environment or more generally with dynamic weightlessness conditions. A weightlessness condition is a result of a dynamic balance of forces acting on an object and exists in space vehicles such as space shuttles, satellites and the International Space Station. A microgravity or near-zero acceleration is the common notation of this weightlessness condition. Because the object is in a dynamically balanced weightlessness environment, it is a prone to any perturbations, no matter how small they are. During a typical space flight, gravity perturbations stem from a variety of sources, which among others include atmospheric drag, solar radiation, aperiodic thrust adjusting to keep the vehicles stay on orbit, pump and other mechanical component operations, astronauts exercises. Figure 1 shows some typical g-jitter signatures that were measured by accelerometers on-board a Space shuttle during its regular flight. Its random nature, both spatial and temporal, is clearly revealed.

These random gravity residuals, though small and with a typical magnitude of $10^{-3} \mathrm{~g}_{0}$ to $10^{-4} \mathrm{~g}_{0}$, combined with the imposed thermal gradients required for solidification, still induce an appreciable convective flow in melt pools, which in turn disrupts the diffusion growth process and thus has detrimental effects on crystal growth in space. While many different means have 
been developed to counter-balance the g-jitter effects, our group has been working on the concept of applying the magnetic damping principle to suppress the g-jitter induced convection in space environment [3-6]. Magnetic damping had long been practiced in materials processing industries to control molten metal feeding and to suppress unwanted thermal convection during crystal growth under terrestrial conditions. Its application to the space environment, however, requires additional assessment. Since magnetic damping originates from different origin than gravity, it is conceptually plausible to combine it with microgravity. However, magnetic damping force is proportional to the velocity field, which is very small indeed in microgravity and natural questions arise on the effectiveness of the application of magnetic damping in space environment and its implementation in space crystal growth systems.

This paper presents our numerical modeling work and ground-based experimental verification or physical modeling of g-jitter induced thermal convection with and without the presence of an external magnetic field. Our numerical model entails both 2-D and 3-D calculations with and without solidification using either fixed grid or moving grid computational methodologies. These calculations are based on our in-house finite element code that has been developed over the past eight years and that is capable of predicting complex multiphysical phenomena associated with a wide range of materials processing systems [6-13]. To validate the model predictions, a ground-based thermal oscillator is developed, where wall temperatures are oscillated to produce an oscillating temperature gradient and transparent fluids are used to facilitate flow visualization. The measurements taken on this physical model are used to check the model predictions obtained from the numerical models. In what follows, the numerical model development and simulations are discussed and the comparison of the model predictions against the experimental measurements of both fluid flow and solidification in physical models is presented. Results show that magnetic damping can be effectively combined with microgravity to provide a viable means for suppressing the unwanted convection or solute spiking induced by g-jitter, thereby a diffusion-controlled crystal growth process is possible. The mathematical model presented, with further validation by experiments on melts in magnetic fields which is being pursued now in our laboratory, should be useful for both fundamental understanding and for designing and interpreting space-based experiments. 


\section{The Mathematical Model}

The usefulness of a mathematical model lies in the fact that once established and validated, it can be applied to study the behavior of a crystal growth system with a wide range of geometric, material and operating parameters. Here we consider a typical Bridgman-Stockbarger single crystal growth system as illustrated in Figure 2. The time dependent phenomena of fluid flows, heat and mass transfer in the above system with the presence of an external magnetic field are described by the Navier-Stokes equations with the time varying gravity force and the Lorentz force resulting from the imposition of a magnetic field. For the melt flow, the standard Boussinesq approximation, $\rho=\rho_{0}\left[1-\beta_{T}\left(T-T_{m 0}\right)-\beta_{C}\left(C-C_{0}\right)\right]$, has been used. Furthermore, the thermoelectric effect, i.e., $\vec{J}=-S \nabla T$, is neglected, because of the lack of reliable data for the absolute thermoelectric power $S$ of the fluid. For the present system, the magnetic Reynolds number is small, so the induced magnetic field maybe neglected in comparison with the imposed magnetic field and the time variation of the induced electric field is also small. These considerations allow us to reduce the Maxwell equations to one equation for the electric potential. With an appropriate choice of scales, the governing equations may be written in the non-dimensional form as follows. For the melt, these equations become:

$$
\begin{aligned}
& \nabla \cdot \vec{U}=0 \\
& \frac{\partial \vec{U}}{\partial t}+\vec{U} \cdot \nabla \vec{U}=-\nabla p+\nabla^{2} \vec{U}-\left[G r_{T}\left(T-T_{m}\right)+G r_{S}(C-1)\right] \vec{g}(t) \\
& +H a^{2}(-\nabla \Phi+\vec{U} \times \vec{B}) \times \vec{B} \\
& \frac{\partial T}{\partial t}+\vec{U} \cdot \nabla T=\frac{1}{\operatorname{Pr}} \nabla^{2} T \\
& \frac{\partial C}{\partial t}+\vec{U} \cdot \nabla C=\frac{1}{S c} \nabla^{2} C \\
& \nabla^{2} \Phi=\nabla \cdot(\vec{B} \times \vec{U})
\end{aligned}
$$


where $\Phi$ is the electric potential.

The crystal and the ampoule move at the constant pulling velocity. The solute diffusion in crystal is also ignored. With these simplifications, the governing equations can be written as follows,

$$
\begin{aligned}
& \frac{\partial T_{c r y}}{\partial t}+V p\left(\hat{e}_{z} \cdot \nabla T_{c r y}\right)=\frac{\kappa_{c r y}}{\kappa} \frac{1}{\operatorname{Pr}} \nabla^{2} T_{c r y} \\
& \frac{\partial T_{a m p}}{\partial t}+V p\left(\hat{e}_{z} \cdot \nabla T_{a m p}\right)=\frac{\kappa_{a m p}}{\kappa} \frac{1}{\operatorname{Pr}} \nabla^{2} T_{a m p}
\end{aligned}
$$

In deriving the above equations, the following scales are used: $R_{0}=0.5 W_{i}$ for length, $U_{0}=v / R_{0}$ for velocity, $t_{0}=R_{0}^{2} / v$ for time, $p_{0}=\rho_{0} U_{0}^{2}$ for pressure, $C_{0}$ for concentration, $g_{0}$ for gravity, $B_{0}$ for magnetic field, $\Delta T=\left(T_{h}-T_{c}\right)$ for temperature while the temperature is non-dimensioned by $\left(T-T_{c}\right) / \Delta T$. The non-dimension parameters are defined as:

$$
\begin{aligned}
& G r_{T}=\beta_{T} R_{0}^{3} g_{0} \Delta T / v^{2}, G r_{S}=\beta_{S} R_{0}^{3} g_{0} C_{0} / v^{2}, \\
& \operatorname{Pr}=v / \kappa, S c=v / D, R a=\varepsilon_{\text {amp }} \sigma R_{0}(\Delta T)^{3} / k_{\text {amp }}, \\
& V_{p}=V_{g} / U_{0}, S t e=H / \Delta T C_{p}, H a=B_{0} R_{0} \sqrt{\sigma_{m} / \rho_{0} v} .
\end{aligned}
$$

The above equations can be solved with appropriate boundary, material and operating conditions. The above equations can be recast into integral forms using the Galerkin Weighted Residuals method and then discretized and solved using the finite element method. The procedures can be found in standard finite element textbooks and our numerical treatment including automatically time step schemes is detailed in other publications $[6,11-13]$ and thus omitted here. As the solidification front is unknown a priori, an iterative procedure is required to determine its location. Both the fixed and moving grid methods have been used, depending on the applications being considered. The former entails the use of enthalpy or effective heat capacity along with flow resistance due to solidifying media, whereas the latter allows the mesh to deform 
to confirm with the fluid flow, thermal and solutal balance conditions along the solidification front.

While the above mathematical formulations are written specifically for the system in Figure 2, relatively a few modifications are required to study other systems. This flexibility is required and is important in that a physical model, often ground-based, is not able to meet the same conditions as the real system but is designed to check certain features of the mathematical model.

\section{Ground-Based Flow and Solidification Experiments}

The experimental system or a physical model for the study of the effect of thermal induced oscillating convection and solidification and to check the mathematical model predictions is schematically sketched in Figure 3. The system consists of three computers, a laser generator, a CCD camera, a test cavity cell, a heat stripe, two isothermal water baths and an expansion board. The function of the computer on the right is to collect temperature data and to control the shutter of the CCD camera for flow visualization and PIV measurements. Two integrated circuit boards (CIO-DAS 1402/16 and CIO-CRT05, both from ComputerBoards Inc.) were installed in the computer, with the former for analog/digital conversion, and the later the control of shutter speed of the CCD camera. The middle computer is to control the heat stripe mounted on the test cell. It uses its own counter board and sends signals to the AC power generator (Model XHR 150-7 from XANTREX Company) to generate the oscillating electrical wave required to produce an oscillating temperature field. The computer on the left is a dual processor PC, designed to perform data intensive computations for image processing. It combines image capturing and image processing into one unit. Its high speed and big memory makes it possible to save the images as fast as needed for our applications. This computer is synchronized with the computer on the right to obtain the temperature and velocity at the same time.

The main part of the system is a rectangular cavity with a dimension of $1.80 \mathrm{~cm} \times 2.03 \mathrm{~cm} \times 15.0 \mathrm{~cm}$. The length is 6 times more than the height and width to eliminated the 3-D effect. The top and bottom walls were made of $1.27 \mathrm{~cm}$ thick acrylic. Also some polystyrene was glued on these plates for insulation purpose. The two sidewalls were made of $3.16 \mathrm{~mm}$ thick grade copper in 
order to get good heat conduction. To prevent the copper side walls from reflecting laser's light, black layer was painted on the inside surface of the sidewalls.

To generate a temperature gradient across the test cell, one vertical side of the cell is set at a constant temperature and the other vertical side at a sine wave oscillation around the average temperature. To do this, circulating water provided by a thermomixer goes through the outside surface of the right wall. This thermomixer can work with a temperature ranging from $-30^{\circ} \sim 100^{\circ}$ C. On the outside surface of the left-side wall a Kapton Flexible Heater (Omega Company) was glued with a wire density of $10 \mathrm{watt} / \mathrm{in}^{2}$. In order to create a sinusoidal temperature at the leftside wall, an electrical power controlled by a signal generated by the function generator with sinusoidal change goes through the wire of the heater. The excess heat flux is removed by circulating water through a chamber attached to the heater, which is connected to another thermostat (B.Braun Instruments) with an operating temperature range of $-50^{\circ} \mathrm{C} \sim 250^{\circ} \mathrm{C}$ and temperature stability of $\pm 0.01^{\circ} \mathrm{C}$.

The Particle Image Velocimetry system used in this experiment included three parts: visualization equipment (Laser), image recording equipment (CCD camera) and analysis software (Insight NT). The Ion Laser (Stabilite 2017) is from Spectra Physics Laser Inc. and Argon is used as an excitation medium. It is an oscillating amplifier of light and this unique light source can output monochromatic, singularly directional and coherent beams. In our case, the direction of the 2 watts continuous laser wave is first turned by a flat mirror and then expanded to a light sheet by a cylindrical mirror.

The Model 630044D PIVCAM 4-30 CCD video camera from TSI offers $748 \times 486$ pixels resolution and has a frame rate as fast as 30 frames per second. Compared with our slow flow in the test cell, this camera has enough high accuracy combined with high image capture rates for time-sequenced measurements. Also it has a terminal to allow control by insight-NT software or external signals. The Insight-NT software provides three acquisition modes to control the camera, focus mode, single frame mode and sequence frame mode. Focus mode is very useful for setting up the experiment, focusing camera and displaying live video. It displays images continuously and refreshed the images at the maximum camera acquisition mode. Single frame 
mode can acquires only one frame at a time. Sequence mode is used to store the images in real experiments for later analysis. A time file is created after sequence storage finishes.

Proper particle seeding of the flow field is very important to produce an accurate description of the fluid motion. First, the seeding particle should have nearly the same density with the fluid it is applied so that it can be neutrally buoyant. Secondly, the concentration of the particles within the fluid is critical. Too much particles within a unit will blur the image and too less particles cannot reflect the true flow precisely. Previous studies [14] have established the adequacy of these seeding conditions for the successful and accurate measurement of the flow velocity in the present test set up. In this respect, the seeding particles used in these experiments are nonspherical nominally distributed $30 \mu \mathrm{m}$ resin particles with a specific gravity of 1.02 . And the concentration of particles in pure water is about $6 \times 10^{-5} \mathrm{~g} / \mathrm{ml}$.

Before any measurements were taken, the water was allowed to flow through the thermobaths (or thermomixers) for about half an hour to establish a steady state. Then the required frequency was tuned on and the heater was activated, while the temperature along two side walls was monitored. Various testing cases showed that after $5 \sim 6$ cycles, the temperature and the fluid flow reached a quasi-steady harmonic motion.

In our case, the lowest frequency used was about $0.01 \mathrm{~Hz}$. That means both the flow and temperature become quasi-steady within 10 minutes, or 6 cycles. To ensure the fully oscillated condition so as to collect data for comparison with the numerical modeling, it was allowed to run more than 30 minutes before starting to collect temperature data and capture the images. With this procedure, it seemed to be difficult to establish a perfect oscillation thermal condition with a frequency equal, to higher than, $0.1 \mathrm{~Hz}$. A higher frequency prevented the heated side wall from generating a larger amplitude of temperature oscillation and thus the flow was thus reduced. These factors combined, high frequency signals seemed to cause difficulty in temperature measurements owing to the limited resolution of the thermocouples used for the experiments.

By fixing the temperatures at the walls, the same system can be used to study the solidification phenomena. Experiments on solidification in an oscillating temperature gradient, however, 
require the change of testing cell designs, which are being considered. Thus, the results on solidification so far are obtained using the fixed temperatures at the walls. Nonetheless, these results provide very useful visualized information on our understanding of flow effects on solidification and also a first phase of check on the mathematical model developed.

For solidification studies, succinonitrile ( $\mathrm{SCN}$ ) is used as a working fluid. It is chosen because of its appropriate thermophysical properties that fit well into the experimental system. The size of the test cell is $6.2 \mathrm{~mm} \times 20 \mathrm{~mm} \times 120 \mathrm{~mm}$ and the cell is made of $1 / 4$ inch quartz glass. In this configuration, the left side wall is fixed at a temperature higher than the melting point and the right hand side is kept at a constant temperature lower than the melting point. The top and bottom walls are adiabatic so that the laser sheet can cut through and illustrates the fluid on its path. The seeding particles are porous silica sphere that are compatible for flow visualization in liquid SCN, and the size of the seeding particles is $70 \mu \mathrm{m}$ in diameter. Since SCN is solid at the room temperature, it is first melted by applying a temperature $1 \mathrm{~K}$ higher than the melting point on both sides of the testing cell. After SCN in the cell became liquid and reached to a uniform temperature within the cell, the temperatures of the thermal baths are adjusted so that the wall temperatures are fixed at $T_{R}=337.15 \mathrm{~K}$ on the right and $T_{L}=328.65 \mathrm{~K}$ on the left. Solidification gradually starts and reaches a steady state after sufficiently long time. Both transient and steady state results were obtained using the experimental system.

\section{Results and Discussions}

With the above mathematical model and experimental system, extensive numerical simulations and experiments were conducted. A selection of these results is presented below, which includes comparison of mathematical model predictions and experiments for both flows and solidification and also 2-D and 3-D model simulations for the flight system as shown in Figure 2. In conducting the numerical simulations, mesh independence studies have been made before collecting the numerical data. For experiments, repeatability tests were also conducted to ensure the accuracy of the experiments. 


\section{Oscillating natural convection}

The numerical model simulations and experimental measurements of transient natural convection induced by oscillating wall temperatures under terrestrial conditions are compared in Figure 4. There is a set of snap shots of the measured and computed results at various instants were depicted for a $45^{\circ}$ tilted configuration, where gravity points downwards (see Figure 4). To ensure one-to-one comparisons, the measured wall temperatures from the physical model are input into the mathematical model, along with no slip wall boundary conditions for velocities, to obtain numerical results. Water is used as it provides a convenient transparent medium for flow visualization. The top and bottom walls of the testing cell were adiabatic. From the experimental measurements and computed results for other conditions as well, it is found that the convection pattern and flow oscillation is a strong function of applied frequency and is also dependent upon the orientation of the liquid cell with respect to gravity. Inspection of Figure 4 illustrates that the measured and computed results for this configuration are in excellent agreement for both flow patterns and velocity magnitudes. The flow field is characterized by three flow cells, with two large ones, approximately equal in size and strength, occupying the entire liquid pool and a small one appearing at the upper corner of the hot wall.

\section{Solidification at constant wall temperatures}

To investigate flow effects on solidification, the testing cell was positioned such that the thermal gradient forms with gravity in various angles. Again, the temperatures measured from the experiments were input into the mathematical model to obtain numerical data for comparisons. Figure 5 shows one such comparison where the thermal gradient is proportional to gravity, which points downward.

Figure 5a shows the experimental result after two frames cross-correlation, which is plotted along with the velocity profile and interface position obtained from numerical simulation in Figures 5b-c. The calculations were carried out using the mathematical model described above with a deforming finite element approach [11]. Examination of these figures shows that the experimental measurements and numerical model predictions are in excellent agreement both qualitatively and quantitatively. Both computed results and experimental measurements show that a single recirculating loop is developed in the liquid pool, which is attributed to the 
buoyancy. The hotter SCN is climbing up along the left wall where temperature is high, while the colder SCN is washing down the solid-liquid interface. Strong convection induced in the pool exerts profound influence on the solidification behavior, as is evident in the interface morphology shown in Figure 5c. In the absence of the convection, the solidification front or interface shape would be flat, which is confirmed by additional numerical simulations using both fixed and moving grids. In the presence of convection, the interface exhibits a non-flat shape such that a thin solid crust exists at the top left corner and the solid layer gradually increases in thickness down along the left wall. In this system, a fluid particle leaves the solid front at the bottom with a relatively colder temperature. It absorbs thermal energy from the high temperature wall and gets hotter as it moves up along the hot wall. At the top of the cell, the fluid particle attains the highest thermal energy but there is forced by mass conservation to be in contact with the cold solidification front. As it travels down along the colder solid-liquid interface, it loses its thermal energy to the solid, which is then conducted through the solid layer to the wall fixed at the lower temperature, thereby causing the interface shape to be thinner at the top and thicker at the bottom, as appears in Figure 5c.

Simulations and experiments for other conditions further indicate that a change in wall thermal conditions and/or in placements of the testing cell results in a changed intensity of convection and hence solidification front morphology. However, the fluid flow pattern remains largely the same, i.e., a single large loop recirculating the entire liquid pool. An exception comes from the case where the cell is tilted $90^{\circ}$ with the hot wall at the bottom. Under this condition, when the Rayleigh's instability condition is met, a completely different interface morphology exists, which is confirmed by both experiments and numerical modeling.

\section{Numerical simulations for the flight system}

With the mathematical model validated as described above, numerical simulations were conducted using appropriate boundary and thermophysical parameters for the system illustrated in Figure 2 for the MEPHISTO-2 and MEPHISTO-4 Space Flight experiments $[1,15]$. Simulations were conducted using the real g-jitter data as shown in Figure 1 and thermal and physical property data are given in [12]. Both 2-D and 3-D simulations were carried out, with the former intended to provide a quicker approximation because the latter requires substantial 
computational resources. The 2-D numerical results are shown in Figure 6. Here the 2-D model is intended to provide an approximation to the middle plane of the 3-D cylindrical model, as the 3-D nature of the g-jitter does not permit the axisymmetry of fluid flow, even though the thermal condition is applied axisymmetrically. While somewhat simplified, the 2-D model allows us to assess some essential features of g-jitter induced flows and solidification behavior. The real $\mathrm{g}$ jitter perturbations are random in direction, orientation and magnitude which cause convective flows in the liquid pool and convection cells developed vary irregularly with time to reflect the nature of the g-jitter driving forces. The spatial convective pattern remains much the same. The dynamic evolutions of the velocity components and concentration near the growth front are selectively shown in Figures 6a-b. The field variables evolve randomly temporally, as expected. The spikes of field variables emerge in response to the spikes of the g-jitter perturbations. These irregular convection signatures are quite obvious and detrimental to diffusion controlling growth. When a magnetic field $\left(B_{0}=0.5 T\right)$ is present, these spikes are substantially damped. An examination of these results further suggests that the applied magnetic field is more effective in high strength velocity spikes. The concentration uniformity along the interface as a result of $\mathrm{g}$ jitter induced convection with and without an imposed magnetic field is compared in Figure 6c, which is measured by $\left(C(t)_{\max }-C(t)_{\min }\right) / C(t)_{\text {average }}$. It transpires that g-jitter has a noticeable, though small, effect on the interface position but may strongly perturb the concentration field near the interface, however an applied magnetic field is able to smooth out the deleterious velocity/concentration spikes caused by the g-jitter disturbances and thus to control the fields at the un-perturbed microgravity condition.

In microgravity environment, the flow structure is truly three-dimensional because of random nature of g-jitter in both space and time, and thus 3-D models are developed to fully appreciate the complex 3-D flow structure. Some of these results obtained from the 3-D model are shown in Figure 7, which correspond to the appearance of the highest spike of the velocity evolution in 2D model. Here the boundary conditions used are the same as for the 2-D model. The flow patterns in the $x-z$ and $y-z$ plane cuts (Fig. 7a-b) clearly reveal the 3-D nature of the flow field. Detailed comparison of the 3-D and 2-D models, however, shows the $y-z$ plane flow pattern is still similar to the 2-D case that uses the same g-jitter data. The corresponding flow patterns with 
an applied magnetic field oriented in the z-direction $\left(B_{0}=0.5 T\right)$ are shown in Figure $7 \mathrm{c}-\mathrm{d}$. These results confirm further that the strong magnetic damping effect can be achieved on the gjitter driven flows. In the $y-z$ plane, the strong flow is substantially reduced and becomes virtually a plug field and the disturbance resulting from g-jitter is almost completely damped out by an applied magnetic field. Figure 8a-b show the z-position and concentration distribution of the 3-D growth interface corresponding to the above flow fields, whereas Figure 8c depicts the time evolution of the concentration uniformity along the growth interface in the absence (upper) and in the presence (lower) of a magnetic field respectively. Apparently, the deleterious effects resulting from the $\mathrm{g}$-jitter induced convection are almost entirely eliminated.

\section{Conclusion Remarks}

This paper has presented a numerical and experimental study of g-jitter induced fluid flows and their effects on solidification under combined action of magnetic fields and microgravity. The 2$\mathrm{D}$ and 3-D numerical models are based on the finite element solution of transport equations governing the transient g-jitter driven fluid flows, heat transfer and solutal transport during solidification with and without an applied magnetic field in space vehicles. To validate the model predictions, a ground-based g-jitter simulator using transparent fluids has been established using the oscillating wall temperatures where timely oscillating fluid flows are measured using a laser PIV system. The measurements are compared well with numerical results obtained from the numerical models. Results show that a combined action derived from magnetic damping and microgravity can be an effective means to control the melt flow and solutal transport in space single crystal growth systems.

\section{Acknowledgments}

Support of this work by NASA (Grant \#s: NCC8-92 and NAG8-1693) and by the U.S. Air Force Office of Research subcontracted through VLOG, Inc (Grant No.: VAN00138704451) is gratefully acknowledged, and so is the assistance of Mr. R. Lentz with the experimental setup and instrumentation. 


\section{$\underline{\text { References }}$}

1. E.S. Nelson, "An Examination of Anticipated G-jitter in Space Station and its Effects on Materials Processes", NASA TM 103775 (1991).

2. N. Govindaraju and B.Q. Li, "A Macro/micro Model for Magnetic Stirring and Microstructure Formation during Solidification", J. Energy Cons. Managnt., Vol. 43, (2002), p. 335-344.

3. B.Q. Li, "G-Jitter Induced Free Convection in a Transverse Magnetic Field," International Journal of Heat and Mass Transfer, Vol. 39, n14, (1996), p. 2853-2860.

4. B.Q. Li, "Effect of Magnetic Fields on Low Frequency Oscillating Natural Convection," International Journal of Engineering Science, v34, n12 (1997), p. 1369-1383.

5. Y. Shu, B.Q. Li, B. Q., and H.C. De Groh, "Magnetic Damping of g-Jitter Induced DoubleDiffusive Convection," Numerical Heat Transfer, Part A, in printing.

6. B. Pan, and B.Q. Li, "Effect of magnetic fields on oscillating mixed convection," International Journal of Heat and Mass Transfer, Vol. 41, n 17, (1998), p. 2705-2710.

7. D. Y. Shang, B.Q. Li and H. C. De Groh, "Magnetic Damping of G-jitter Driven Flows: 3-D Calculations", J. Jpn. Soc. Microgravity Appl., Vol. 15, Supplement II (1998), p. 108-113.

8. B.Q. Li, "Stability of Modulated-Gravity-Induced Thermal Convection in a Magnetic Field", Physical Review E, Vol. 63, (2001), p. 041508-1-041508-9.

9. B.Q. Li and R. He, "Computational Modeling of Electrodynamic and Transport Phenomena under Terrestrial and Microgravity Conditions," Int. J. of Applied Electromagnetics \& Mech., in printing.

10. J. Honda, C. Zhang, B. Q. Li and H. C. de Groh, "A 3-d Model for Magntic Damping of Gjitter Induced Convection and Solutal Transport in a Simplified Bridgman Configuration", Proceedings of the ASME Heat Transfer Division, New York, NY, Nov. 2001.

11 S.P. Song and B.Q. Li, "Surface Deformation and Marangoni Flow in Electrostatically Levitated Droplets", Int. J. Heat Mass Transfer, Vol. 43(2000), p. 3589-3606.

12. K. Li, B.Q. Li and H. C. de Groh, "Effects of Magnetic Fields on G-jitter Induced Convection and Solute Striation during the Melt Growth of Single Crystals", Int. J. Heat Mass Trans., Submitted.

13. K. Li, B.Q. Li and H. C. de Groh, "A Numerical Analysis of Double Diffusive Convection and Solidification under the Combined Influence of Microgravity and Magnetic Fields", Journal of Thermophysics and Heat Transfer, submitted. 
14. Higgins, M. L., 2000, "PIV Study of Oscillating Natural Convection Flow within a Horizontal Rectangular Enclosure," M. S. Thesis, Washington State University, Pullman, WA. 15. C. Benjapiyaporn, V. Timchenko, E. Leonardi, G.De Vahl Davis and H. C. De Groh III . Effects of space environment on flow and concentration during directional solidification, NASA TM 209293 (2000). 


\section{Figure Captions}

Figure 1. The g-jitter signatures (a) x-direction (b) y-direction (c) z-direction taken by an onboard accelerometer during a space flight.

Figure 2. Schematic representation of the 2-D model (also the r-z plane in the 3-D model) for the Bridgman-Stockbarger single crystal growth system in microgravity.

Figure 3. Schematic of the experimental setup for the PIV measurement of thermally induced convection oscillation in a cavity.

Figure 4. Comparison of transient development of oscillating velocity profiles measured by the PIV system and by the finite element model for the configuration tilted at $45^{\circ}$ in the clockwise direction. The applied frequency is $0.025 \mathrm{~Hz}$ and the hot wall is on top. (a1, b1, c1) are measured and $(\mathrm{a} 2, \mathrm{~b} 2, \mathrm{c} 2)$ are numerical results.

Figure 5. Solidification of SCN in a vertical cell, as measured by the PIV system and computed by numerical model. The dimension of the cell is $6.2 \mathrm{~mm} \times 20.0 \mathrm{~mm}$. (a) is the experimental results from INSIGHT-NT software. (b) is the velocity profile from numerical simulation and (c) is the interface positions from numerical model. $T_{R}=337.15 \mathrm{~K}, T_{L}=328.65 \mathrm{~K} . \mathrm{V}_{\max }$ (experiment) $=1.115 \mathrm{~mm} / \mathrm{s}, \mathrm{V}_{\max }$ (computation) $=1.307 \mathrm{~mm} / \mathrm{s}$.

Figure 6. The magnetic damping effects on the time evolutions of (a) the velocity at $x=3.6$, $y=0.5$, (b) the concentration at $x=3.6, y=1.3$ and (c) the concentration uniformity measured by $\left(C(t)_{\max }-C(t)_{\min }\right) / C(t)_{\text {average }}$ along the interface caused by real g-jitter data.

Figure 7. The 3-D flow fields caused by real g-jitter data at time $(t=1.734)$ in the absence of magnetic field (a) $\mathrm{x}-\mathrm{z}$ plane (b) $\mathrm{y}-\mathrm{z}$ plane with $U_{\max }=0.2606$ and in the presence of magnetic field with (c) $x-z$ plane and (d) $y-z$ plane with $U_{\max }=0.1670$.

Figure 8. (a) The z-position and (b) concentration of the 3-D growth interface at time ( $t=1.734$ ) and the (c) the time evolution of the concentration uniformity along the growth interface, which is measured by $\left(C(t)_{\max }-C(t)_{\min }\right) / C(t)_{\text {average }}$ in absence of the magnetic field (upper) and in the presence of the magnetic field (lower) respectively. 

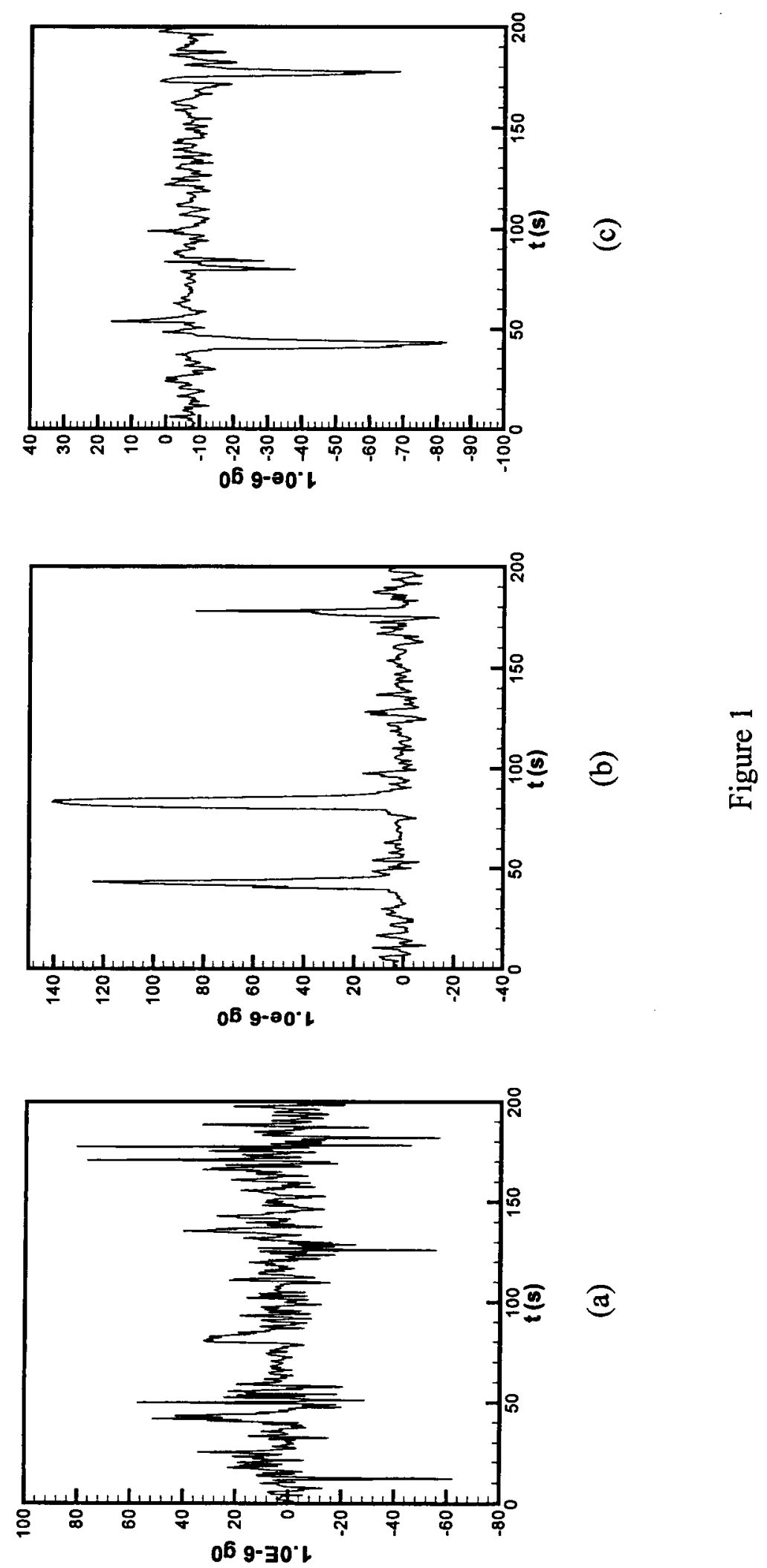


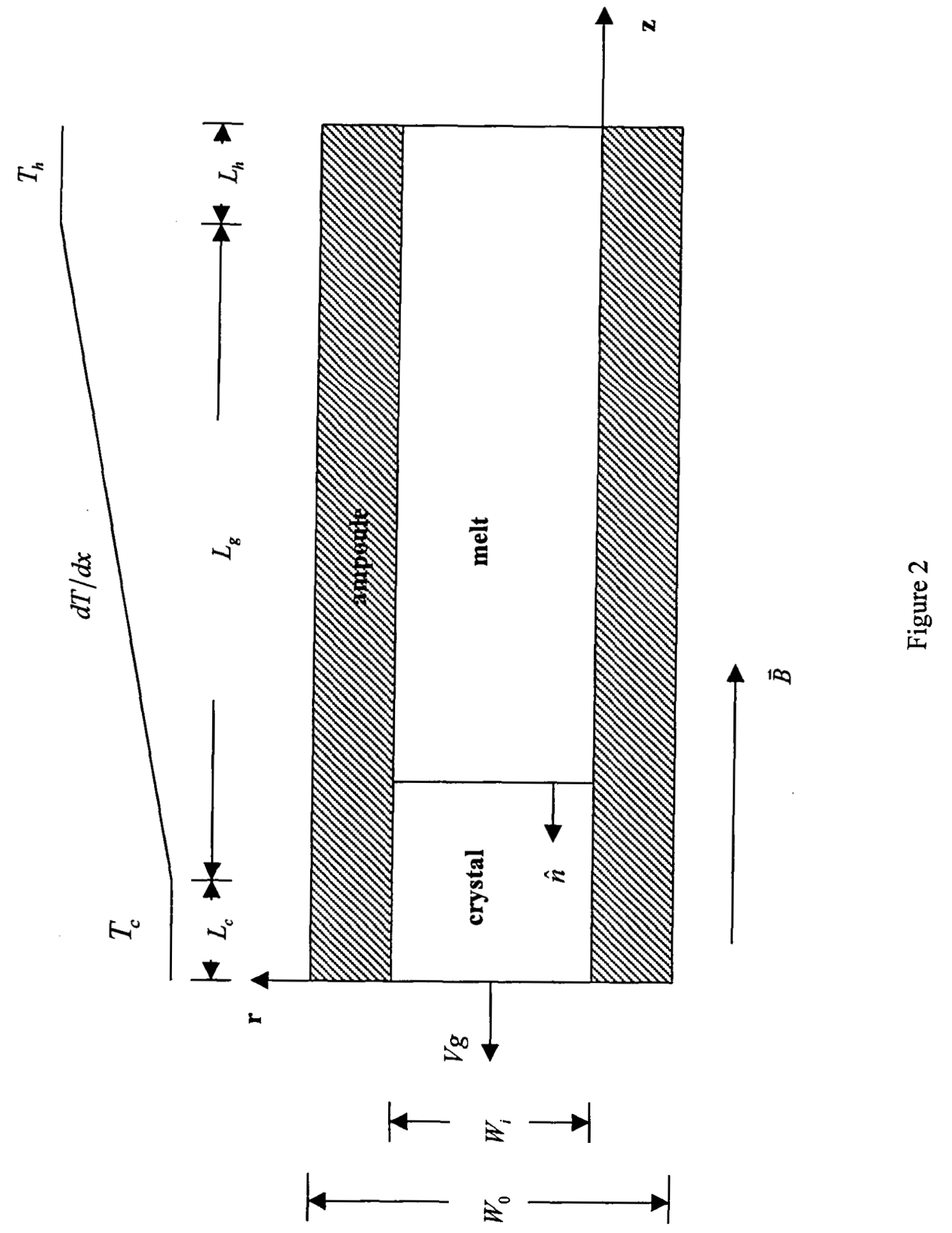




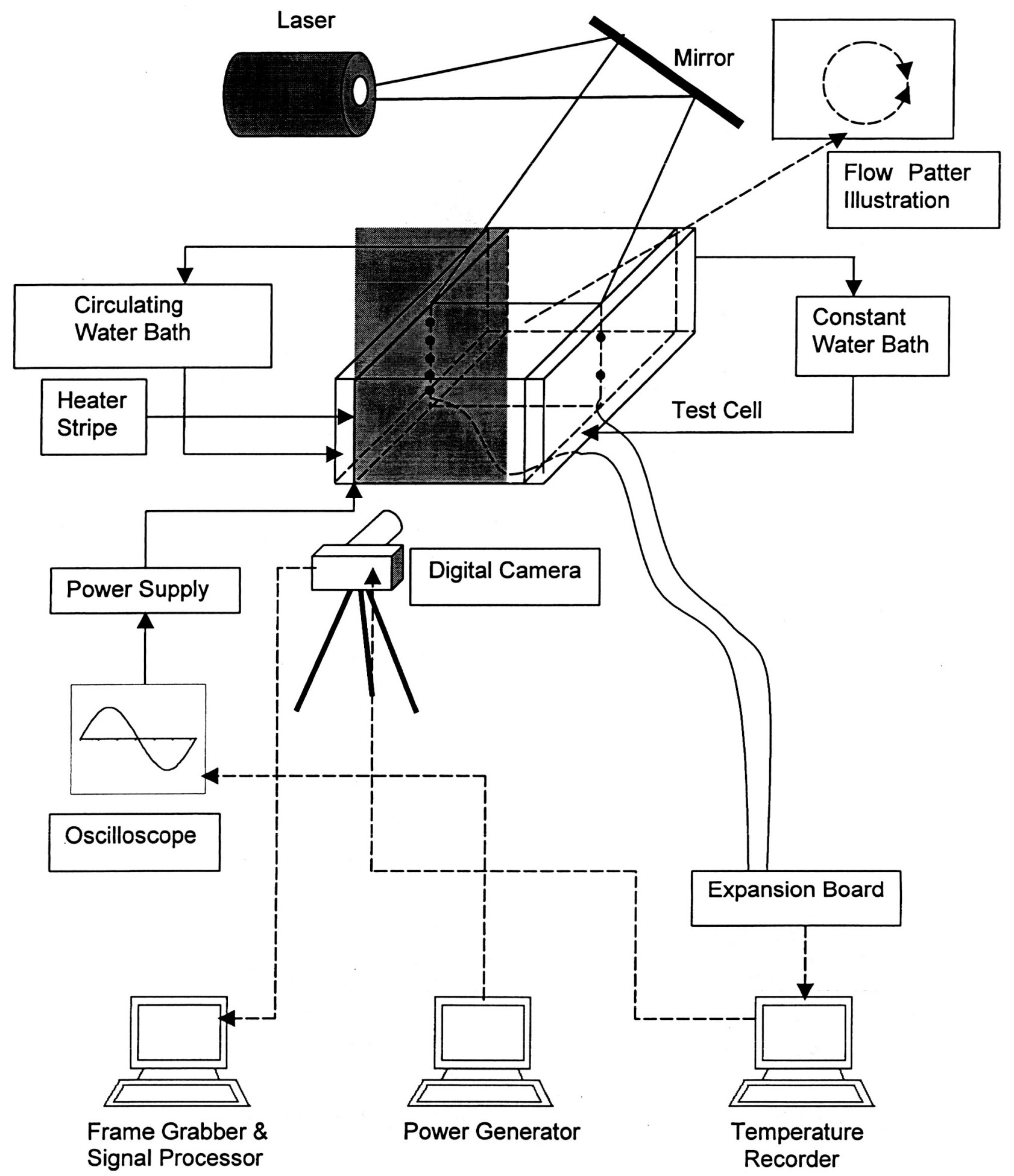

Figure 3 


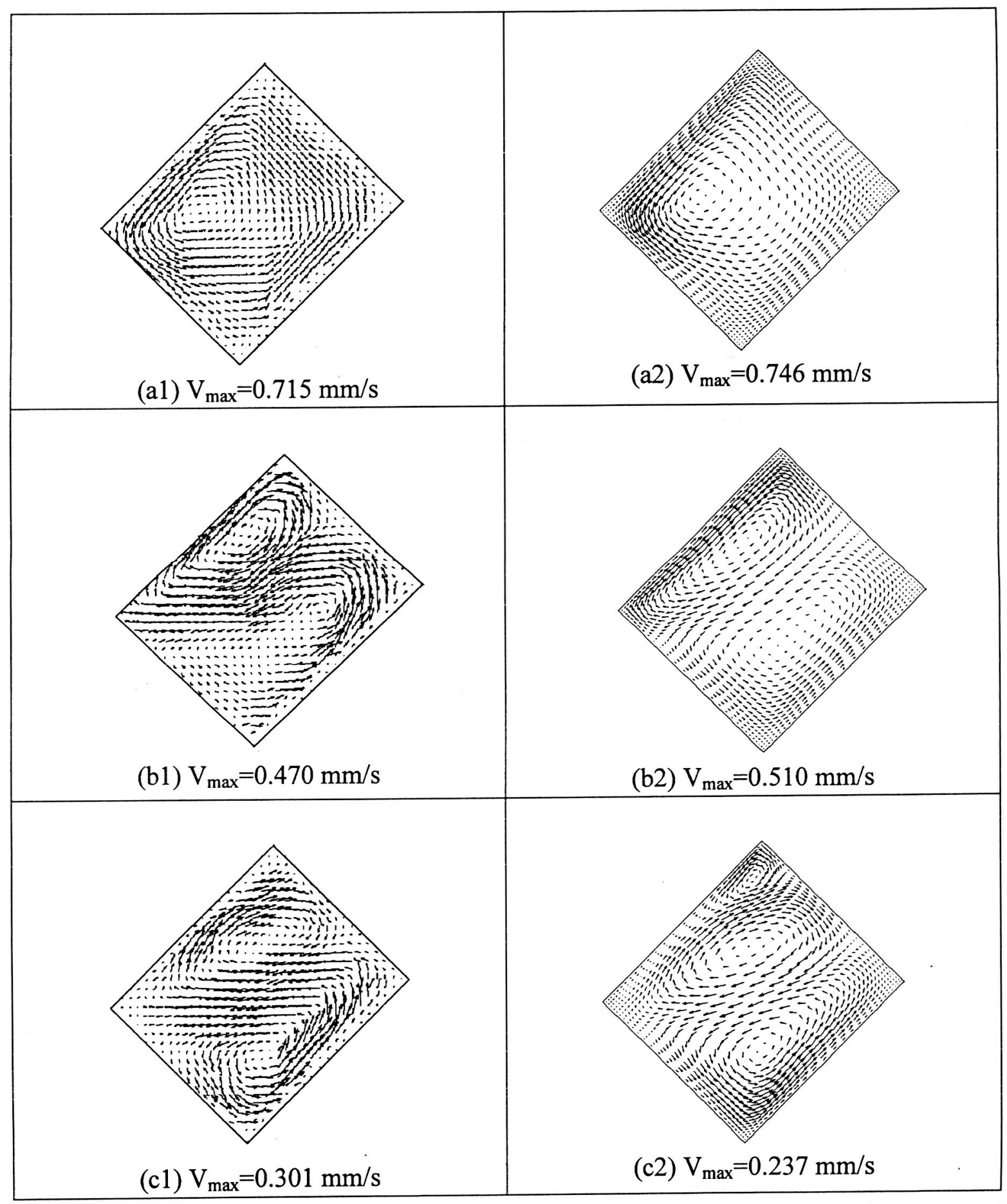

Figure 4 


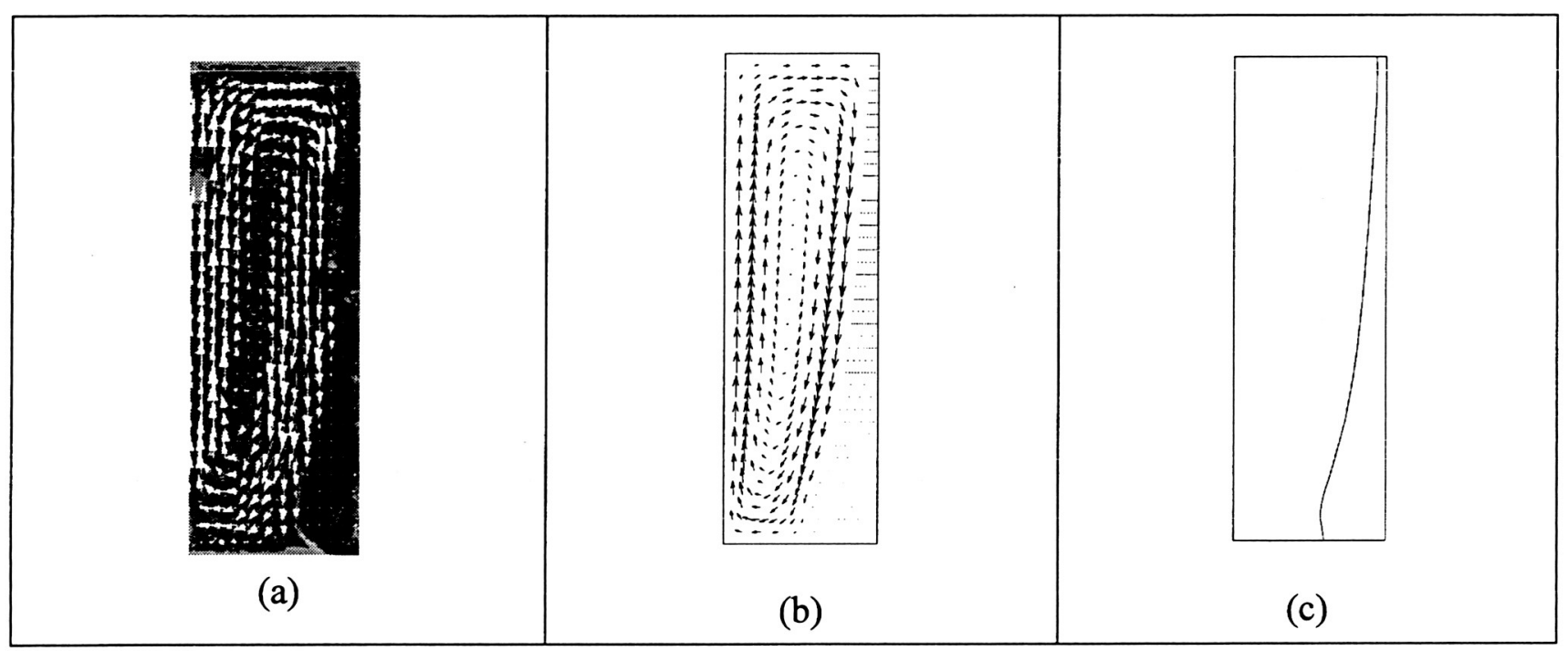

Figure 5 

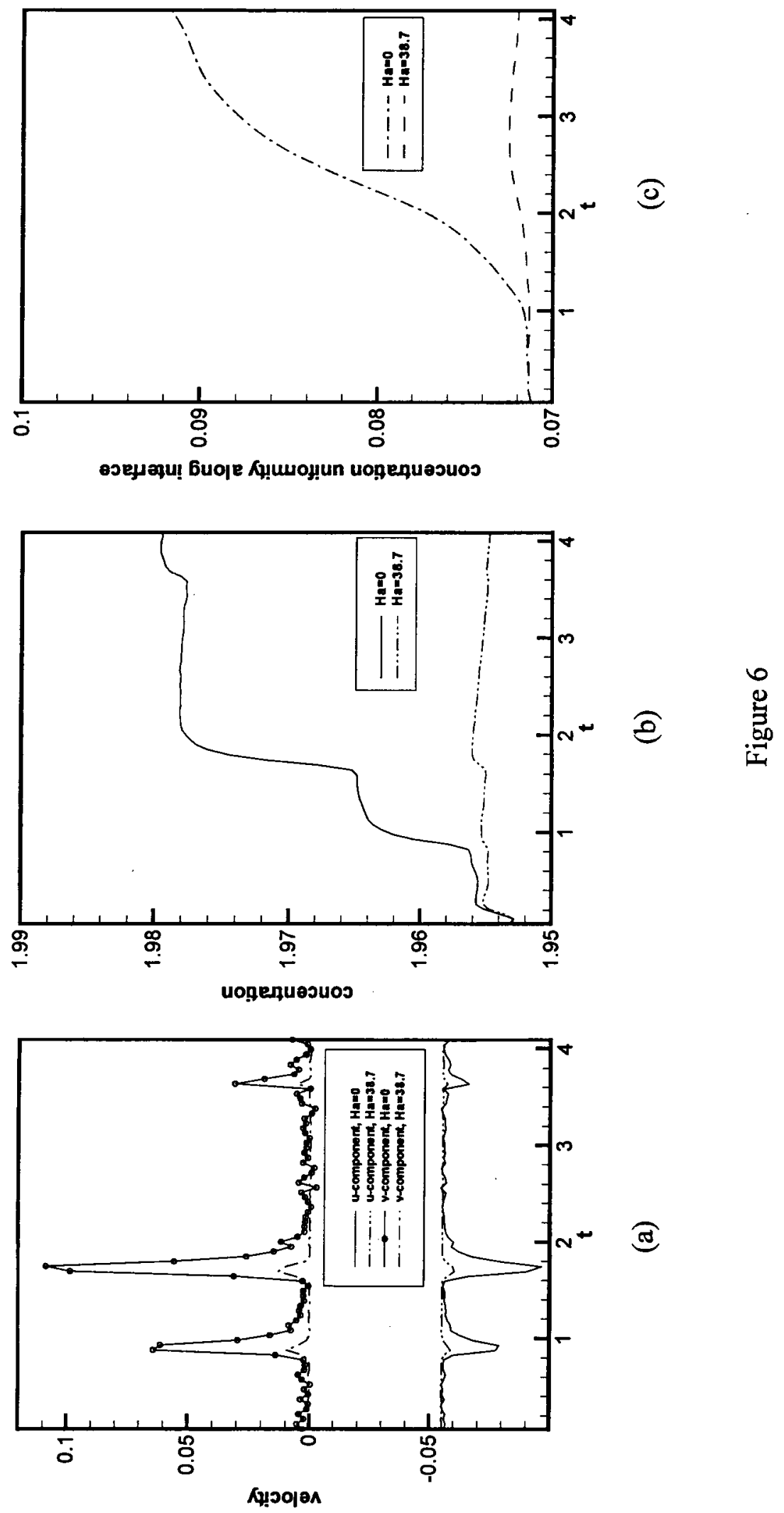


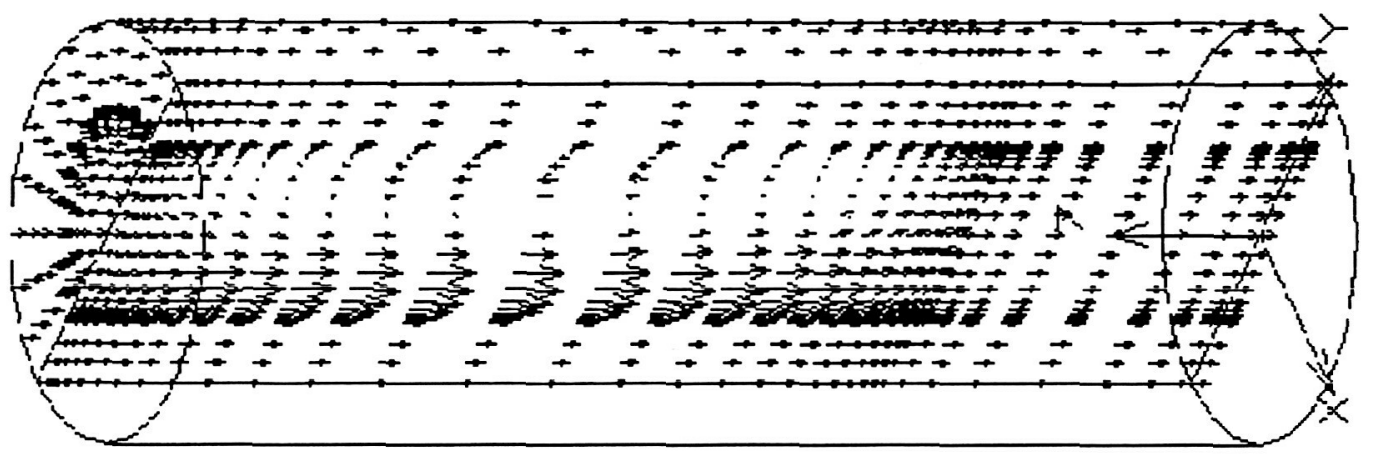

。
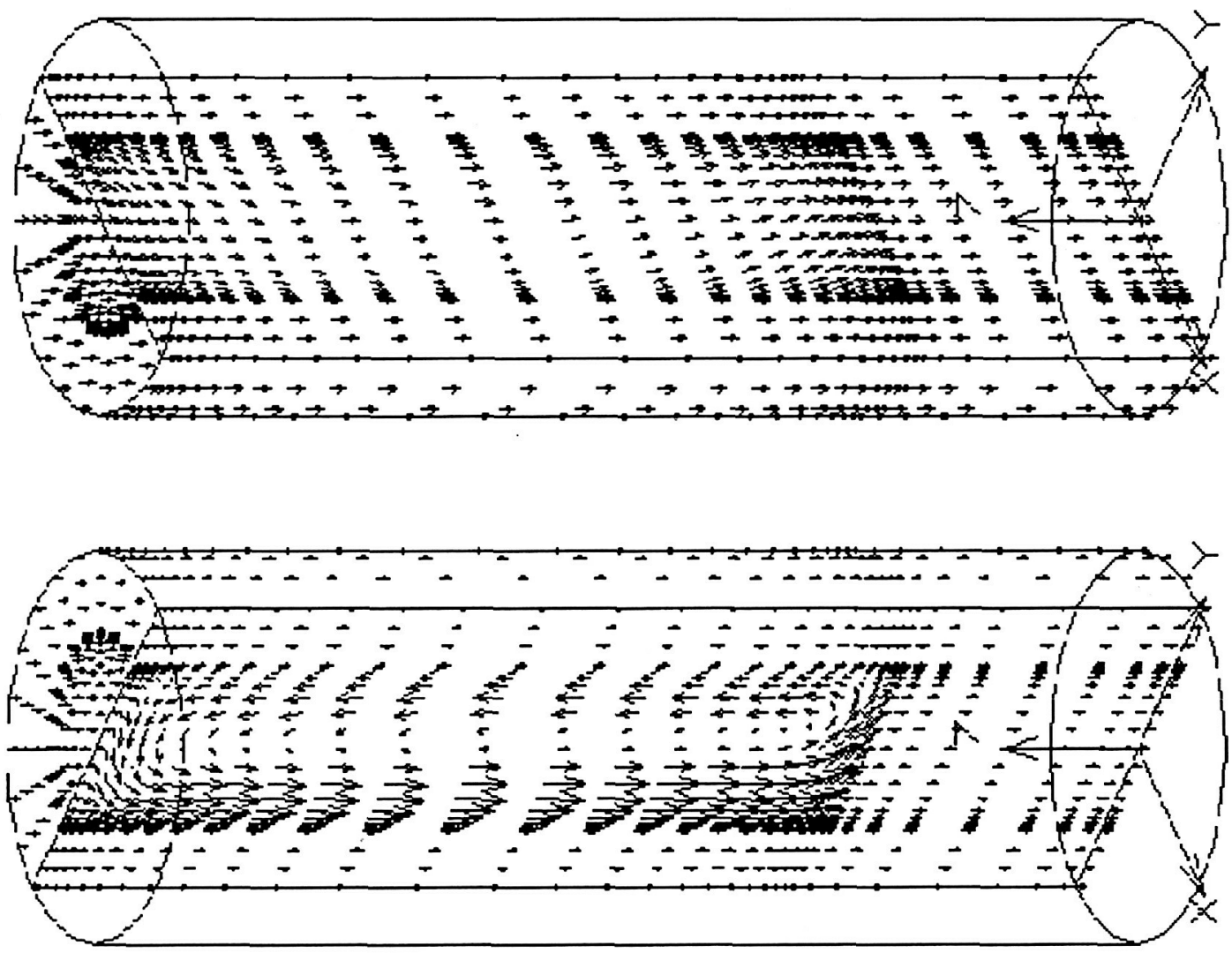

N

奇

อ

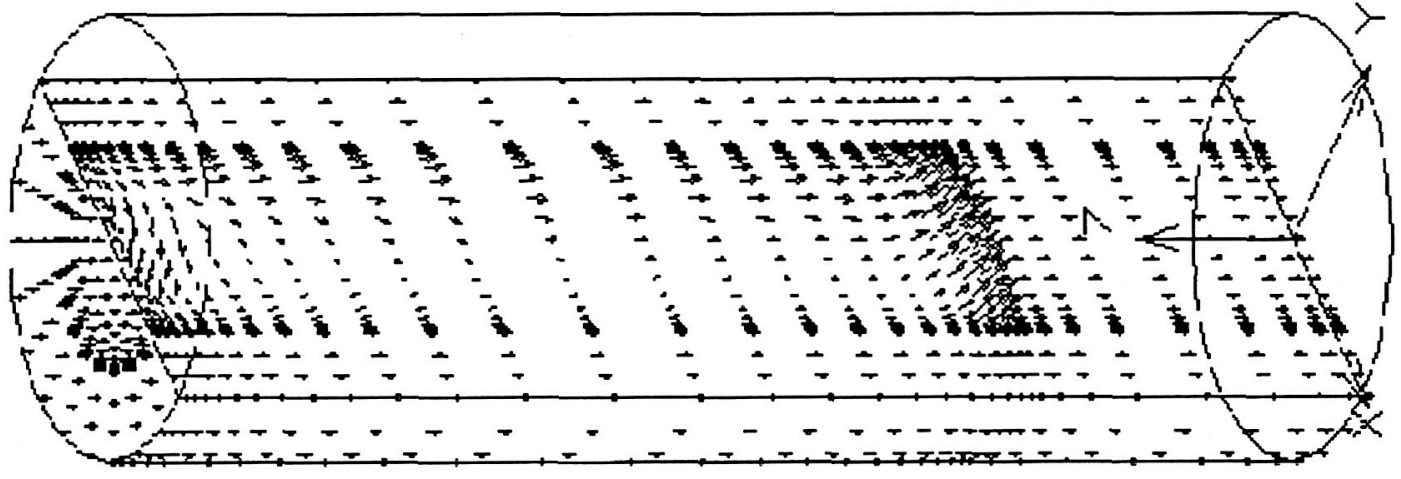



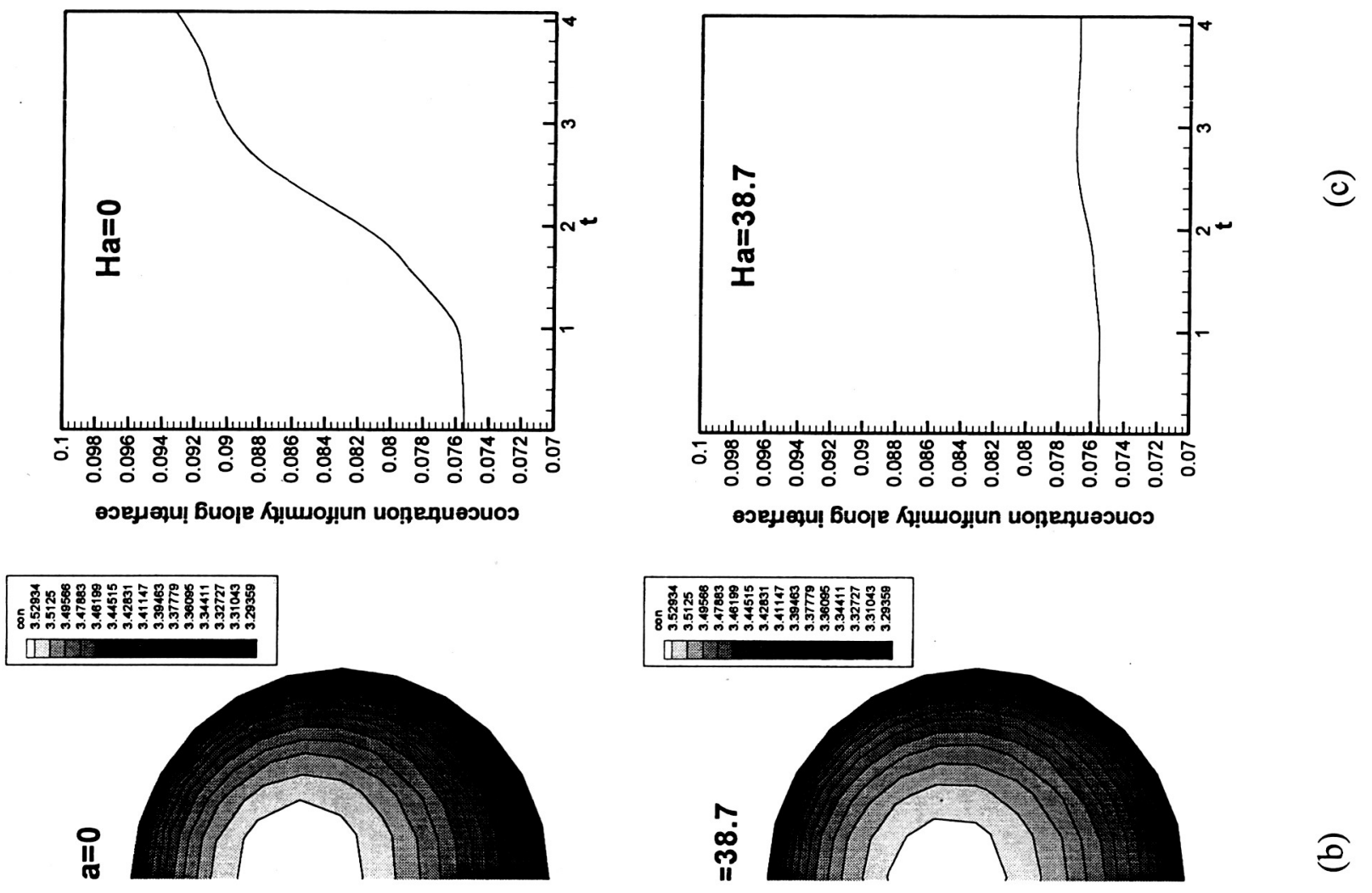

อ 